\title{
PREDICTION AND STUDY OF POLLUTANT DISPERSION IN A STREET- CANYON IN LONDON USING COMPUTATIONAL FLUID DYNAMICS TECHNIQUES
}

\author{
A. GALANI ${ }^{1}$ \\ P. NEOFYTOU, ${ }^{2, *}$ \\ A. VENETSANOS ${ }^{1}$ \\ J. BARTZIS ${ }^{1}$ \\ S. NEVILLE ${ }^{3}$
}

\author{
${ }^{1}$ Environmental Research Lab., INT-RP, NCSR Demokritos \\ Aghia Paraskevi, 15310 Athens, Greece \\ ${ }^{2}$ Thermal-Hydraulics \& Multiphase Flow Lab., INT-RP \\ NCSR Demokritos, Aghia Paraskevi, 15310 Athens, Greece \\ ${ }^{3}$ Westminster Council House, Marylebone Road, London \\ NW1 5PT, United Kingdom
}

Received: 08/07/08

Accepted: 14/04/09 *to whom all correspondence should be addressed: e-mail: panosn@ipta.demokritos.gr

\begin{abstract}
Pollution levels in an urban street-canyon area are determined numerically as part of the European research project OSCAR using the ADREA-HF code. Aim of the modeling is twofold: (i) to investigate the flow-field and carbon monoxide (CO) concentrations in the area and (ii) compare with measurements. The latter is achieved due to the availability of measurements, contrary to previous street-canyon-simulation studies where ADREA-HF was used and where no measurements were available. Results show a tendency of overprediction of $\mathrm{CO}$ concentration by the model that is attributed mostly to the uncertainty of the meteorological data and emission levels within the studied time frames. The concentration distribution and flow field within the canyon are shown to be highly correlated whereas the incanyon induced vortex plays a prominent role in the concentration dispersion.
\end{abstract}

KEYWORDS: Pollutant, Dispersion, Street-Canyon, Computational Fluid Dynamics.

\section{INTRODUCTION}

Dispersion of pollutants originating from traffic is related to the geometry of the urban area and to the traffic conditions. Computational Fluid Dynamics (CFD) modeling is an important tool to understand and assess the airflow and dispersion of pollutants among complex urban geometries. Such model takes into account meteorological conditions, building designs and proximity of roadways to pollutant sources and enables more accurate predictions of pollutant dispersion. Furthermore, the limit of pollutant levels set by the World Health Organisation has led to increased research activity to specify the influence of car emissions on the air quality in urban street canyons. Urban street-canyons consist of uniform parallel building complexes on either side of the street and induce flow recirculations and/or stagnant conditions thus prohibiting the dispersion of pollutants away from inhabited areas.

Vardoulakis et al. (2003) reviewed and evaluated several CFD methods applied in meteorological, wind-tunnel and street canyon studies. In addition, Walton et al. (2002) pursued Large-Eddy Simulations (LES) of the mean flow and turbulence in cubic street canyons. Their results show good agreement between simulations and experimental data. Finally, CFD computations using the ADREA-HF code (Bartzis, 1991) have been carried out by Neofytou et al. (2006a) in order to study the dispersion of the pollution in a street canyon under different wind directions.

The current study is carried out in the framework of the Optimised Expert System for Conducting Environmental Assessment of Urban Road Traffic (OSCAR) project. This project aspires to assess the environmental impact of road traffic in terms of traffic flows, emissions and air pollution. A combined emission measurement and model-prediction campaign was 
carried out in Marylebone Road in London, United Kingdom in 2003-2004. Carbon monoxide (CO), nitrogen monoxide (NO), nitrogen dioxide $\left(\mathrm{NO}_{2}\right)$, nitrogen oxides $\left(\mathrm{NO}_{x}=\mathrm{NO}, \mathrm{NO}_{2}\right)$ and sulphur dioxide $\left(\mathrm{SO}_{2}\right)$ concentrations have been measured at selected time periods whereas the wind directions and wind speeds have been continuously measured at rooftop level. Emission data during the time periods under investigation are also provided. The numerical predictions are performed using the CFD code ADREA-HF (Bartzis, 1991), which also has been used in the past for environmental flow predictions (Neofytou et al., 2006b; Venetsanos et al., 2000).

\section{METHODOLOGY}

\subsection{Measurements}

The monitoring campaign in Marylebone Road was conducted in 2003 - 2004 (01.01.2003 31.12.2004), in order to provide comprehensive air quality databases. The street-canyon has an aspect ratio (Average Height/Width) approximately equal to 1:2. The monitoring site is located in a purpose built cabin on Marylebone Road opposite Mme Tussaud's. The sampling point is located at a height of $3 \mathrm{~m}$, around $1 \mathrm{~m}$ from the kerbside. Traffic flows of over 80,000 vehicles per day pass the site on six lanes. Air quality measurements and on-site electronic traffic counts were conducted throughout the campaign. CO concentrations, wind-direction, wind-speed and emission data were available with the time resolution of one hour. The emission factors were assessed by the Department for the Environment, Food and Rural Affairs.

\subsection{Numerical Method}

The methodology consisted in solving the transient, Reynolds averaged, mass and momentum 3D conservation equations for the mean flow and the mass fraction conservation equation for the pollutant dispersion, until steady state conditions were reached. Boundary conditions for the problem were zero gradient and given value for the inflow boundaries, zero gradient for the outflow boundaries, wall functions for velocities at the buildings surfaces and ground and finally zero vertical velocity at the top of domain.

The computational domain that includes all buildings in the area surrounding Marylebone Road was constructed using actual coordinates provided by the Westminster Council House is shown in Figure 1. It covers an area of $1000 \times 940 \times 200 \mathrm{~m}$ discretised by $70 \times 70 \times 35$ grid cells, which are refined near the measurement location in order to more accurately capture the wind field and concentration distribution.

With regard to the comparison with measurements, two different datasets were selected for the pollutant under consideration, both covering a 2 hour time period. Each dataset provides values for wind speed, wind direction and concentration every hour. Higher accuracy requirements imposed the development of a Fortran 90 code in order to select the proper datasets. This selection was based on the data standard deviation, which should not exceed the values of 5.0, 0.2, 12 and 2.0 for wind direction, wind speed, traffic count and traffic speed, correspondingly. Hence, from each retained dataset the parameters presented small variability during the selected time periods and the mean value was derived for each parameter. The aforementioned values for wind speed and direction were used as input data to the model in order to simulate the pollutant concentrations, which are then compared with the observations. In addition, the source of pollution which is principally the emissions from the cars passing Marylebone Road (Fig. 1) was modelled for each dataset as homogeneous and constant source along the street. 


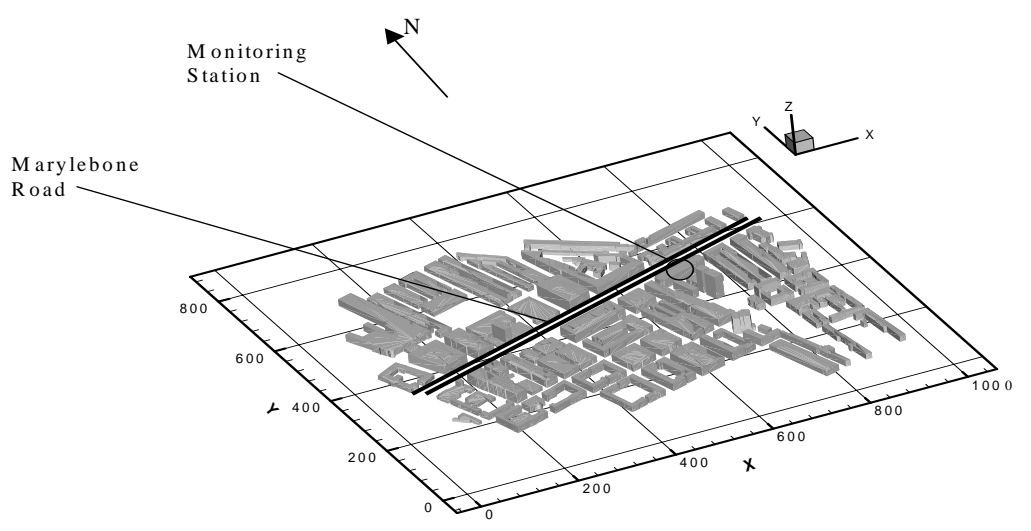

Figure 1. Computational domain of the Marylebone Road area

\section{RESULTS AND DISCUSSION}

The datasets selected and the corresponding mean values for the reported wind speed (WS), wind direction (WD) and street level concentration of CO (SLC in $\mathrm{mg} \mathrm{m}^{-3}$ ) correspond to weekdays and are shown in Table 1. The background concentration values of $\mathrm{CO}$ (BC in $\mathrm{mg}$ $\mathrm{m}^{-3}$ ) correspond to reported annual mean values. The point where the measurements are taken corresponds to $x=727.3 \mathrm{~m}, y=508.7 \mathrm{~m}, z=3 \mathrm{~m}$ of the computational domain. Although ADREA-HF has a tendency to overpredict the measurements, it follows their trend i.e. the ratio of the predicted (SLC prediction$)$ to the observed $\left(S L C_{\text {observed }}\right)$ values is of the same order of magnitude for both cases. The most important factors of uncertainty are the definition of the source of emissions and the reported wind speed and wind direction mean values. These aforementioned data are given on hourly basis. However, their value most probably exhibit substantial fluctuations within each hour. In addition, the background observed concentration values used in the model correspond to annual mean levels.

Table 1. Overview of datasets and numerical predictions for CO street level concentrations (SLC)

\begin{tabular}{cccccccc}
\hline $\begin{array}{c}\text { Dataset/ } \\
\text { Case }\end{array}$ & Date & $\begin{array}{c}\text { Time } \\
\text { frame }\end{array}$ & $\begin{array}{c}\text { Reported } \\
\text { WS } \\
\left(\mathrm{m} \mathrm{s}^{-1}\right)\end{array}$ & $\begin{array}{c}\text { Reported } \\
\text { WD } \\
(\mathrm{deg})\end{array}$ & $\begin{array}{c}\mathrm{BC} \\
\left(\mathrm{mg} \mathrm{m}^{-3}\right)\end{array}$ & $\begin{array}{c}\mathrm{SLC}_{\text {observed }} \\
\left(\mathrm{mg} \mathrm{m}^{-3}\right)\end{array}$ & $\begin{array}{c}\mathrm{SLC}_{\text {prediction }} \\
\left(\mathrm{mg} \mathrm{m}^{-3}\right)\end{array}$ \\
\hline 1 & 17.2 .20 & $11: 00-$ & 1.37 & 211.6 & 0.44 & 0.90 & 1.27 \\
\hline 2 & 19.4 .20 & $15: 00-$ & 3.53 & 92.8 & 0.44 & 0.53 & 0.58 \\
\hline
\end{tabular}

It is very interesting though to see how the concentration distribution is behaving with respect to the flow field. The comparison is carried out between datasets 1 and 2, for which the same source of emissions is assumed. For the height of the measurement point $(z=3 \mathrm{~m})$ the simulated concentration distribution is illustrated in Figure 2. In order to represent the concentration field, a limit of $0.18 \mathrm{mg} \mathrm{CO} \mathrm{m}^{-3}$ of air was set, below which concentration values are not shown. First, it can be seen that Marylebone Road has major contribution to the pollution in its vicinity, which can be attributed to the fact that this street has more traffic and thus more prominent pollution compared to its neighbouring ones.

Furthermore, it can be seen that the concentration levels are higher for case 1 compared to case 2 at $z=3 m$ despite the fact that emission rates are higher than those in case 1 . This can be attributed to relatively higher wind speeds within the street-canyon at that height (Fig. 3) and therefore the more intense dispersion for case 2. Intense dispersion is caused not only from the higher free-stream velocity for case 2 , which results in more marked street-canyon effects, but also from the fact that the wind direction, which is for this case almost aligned with Marylebone Road, forces wind to move towards the west side of the street thus causing pollutant attenuation along $x$-axis. 


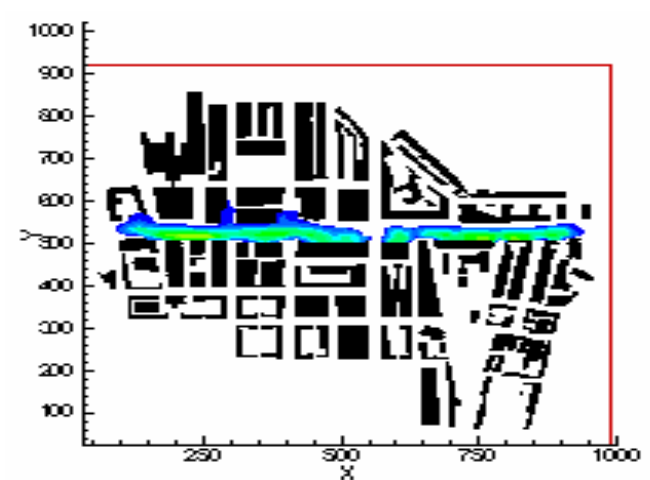

(a)

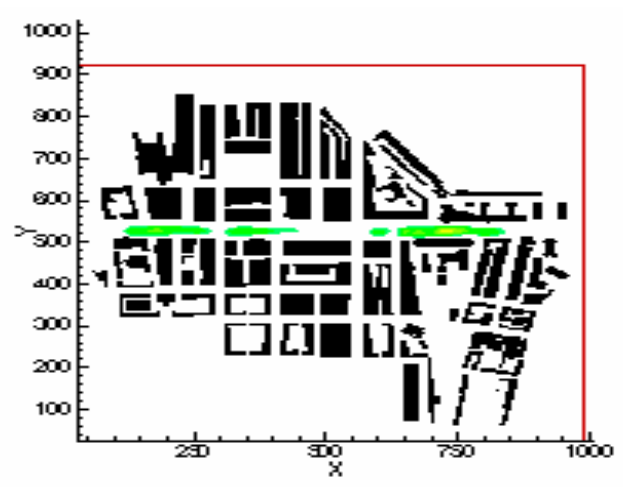

(b)

Figure 2. Simulated concentration distribution for $\mathrm{CO}$ at $\mathrm{z}=3 \mathrm{~m}$ for cases (a) 1 ; (b) 2

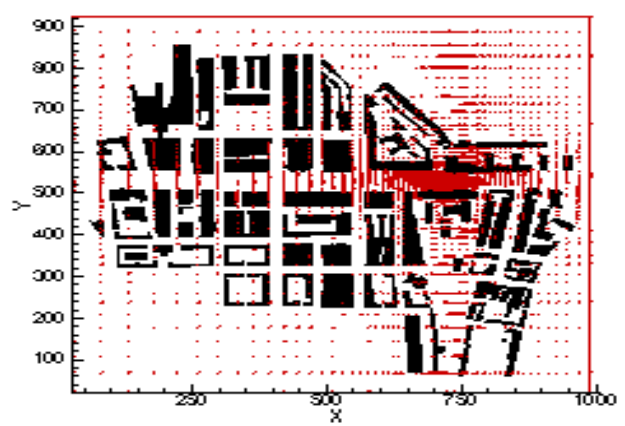

(a)

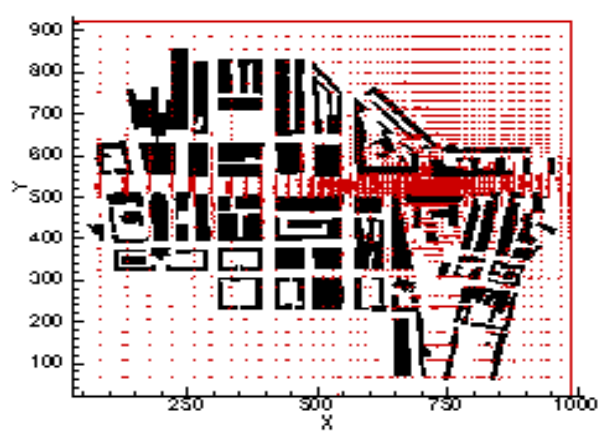

(b)

Figure 3. Simulated wind-velocity field at $z=3 \mathrm{~m}$ for cases: (a) 1 ; (b) 2

In both cases 1 and 2, the existence of a street-canyon vortex can explain the calculated upwind accumulation of pollutant concentration within the canyon depicted in Fig. 2. Although velocities within the canyon are higher than at the crossing of Marylebone Road with Baker Street (Fig. 3), the street-canyon vortex is responsible for maintaining higher in-canyon concentrations than at the crossing, where the concentration is much lower, despite the similarity of the emissions at both locations. Therefore higher velocities do not necessarily result in higher dispersion rates. Figures 4 and 5 present the simulated $\mathrm{CO}$ concentration distribution and velocity field for dataset 1 at three lateral planes along the z-axis, correspondingly. The first height is at $z=1.70 \mathrm{~m}$, which is the population average height. There, concentration levels are much higher than at the other heights $(z=19.87 \mathrm{~m}$ and $z=70.55 \mathrm{~m}$, buildings average and maximum heights, respectively). It is observed that as the height increases, the wind speed increases too, thus causing more intensive dispersion and consequently a decrease in pollutant concentration levels. As shown in figure 4c, at $70.55 \mathrm{~m}$ height, the pollutant is simulated to accumulate substantially in a small region in front of the two highest buildings of the complex. This implies that the height of the buildings is another important factor that influences pollutant dispersion. A similar behaviour is observed for dataset 2. 


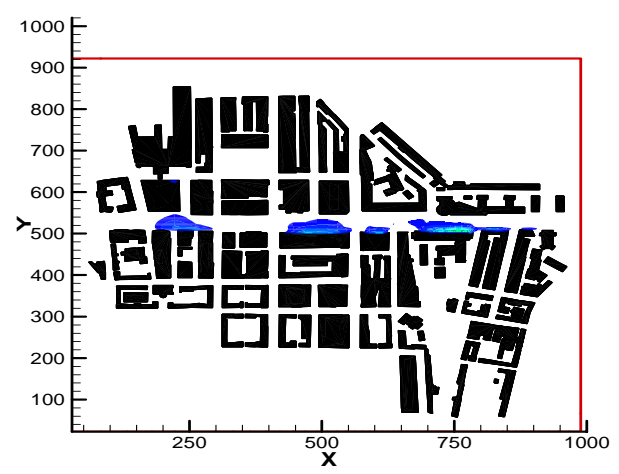

(a)

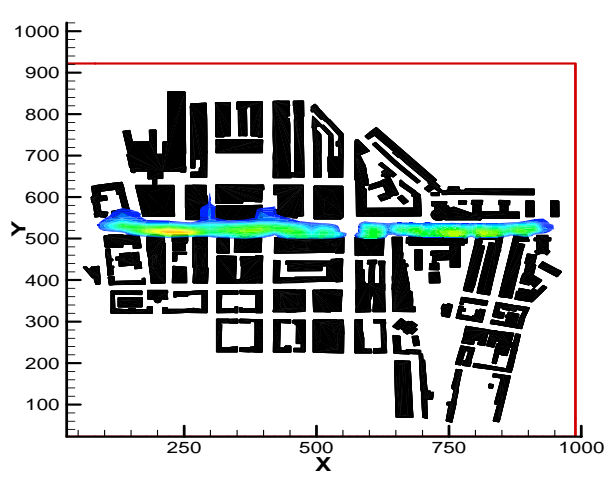

(b)

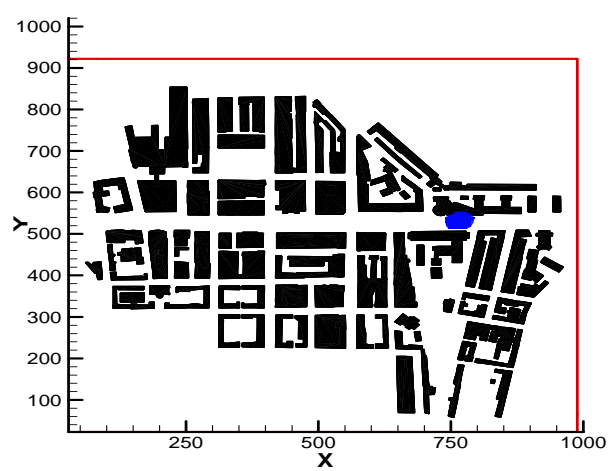

(c)

Figure 4. Simulated CO concentration distribution for dataset 1 at (a) $z=1.70 \mathrm{~m}$; (b) $z=19.87 \mathrm{~m}$; (c) $z=70.55 \mathrm{~m}$

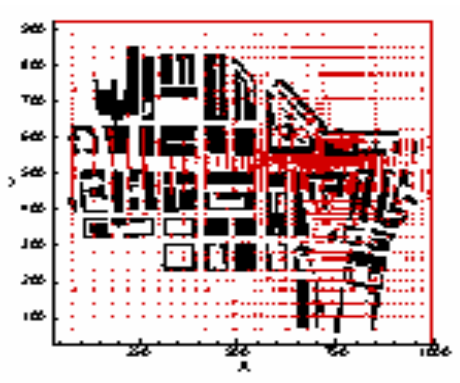

(a)

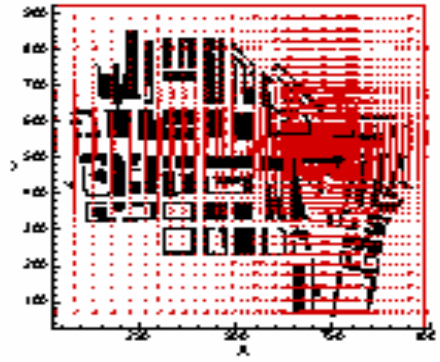

(b)

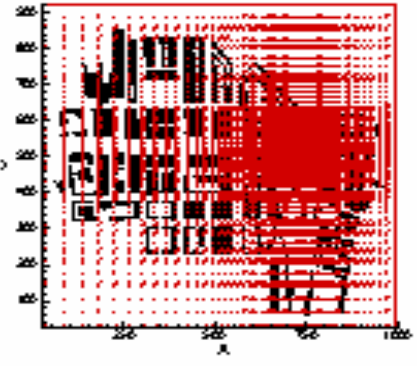

(c)

Figure 5. Simulated wind-velocity field for dataset 1 at (a) $z=1.70 \mathrm{~m}$; (b) $z=19.87 \mathrm{~m}$;

(c) $z=70.55 \mathrm{~m}$

\section{CONCLUSIONS}

Pollution-dispersion modelling in an urban area was carried using the ADREA-HF code. A comparison between observed pollutant levels and simulations was subsequently carried out. This also allowed an assessment of the model, a feature not included in earlier ADREA-HF street-canyon-simulation studies (Neofytou et al., 2006a) due to the fact that measurements were not available. The model tends to over predict the $\mathrm{CO}$ concentrations mainly because of the uncertainty in both the meteorological data and the emissions for the specific time frames considered here. The presence of a street-canyon vortex can explain the observed upwind accumulation of the concentration within the canyon. Furthermore, the wind speed and direction as well as the height of the buildings determine in a major degree the dispersion mechanism. Finally, the wind velocity increase with height causes a decrease in the pollutant concentration levels. 


\section{ACKNOWLEDGEMENTS}

The financial support from the European Union under the contract EVK4-CT-2002-00083 is gratefully acknowledged.

\section{REFERENCES}

1. Bartzis J.G. (1991) ADREA-HF: A three-dimensional finite volume code for vapor cloud dispersion in complex terrain, EUR report $13580 \mathrm{EN}$.

2. Neofytou P., Venetsanos A.G., Rafailidis S. and Bartzis J.G. (2006a) Numerical Investigation of the Pollution Dispersion in an Urban Street-Canyon, Environ. Modell. Softw., 21, 525-531.

3. Neofytou P., Venetsanos A.G.,Vlachogiannis D., Bartzis J.G. and Scaperdas A. (2006b) CFD Simulations of the Wind Environment around an Airport Terminal Building, Environ. Modell. Softw., 21, 520-524.

4. Vardoulakis S., Fisher B.E.A., Pericleous K. and Gonzalez-Flesca N. (2003) Modelling air quality in street canyons: a review, Atmos. Environ., 37, 155-182.

5. Venetsanos A.G., Bartzis J.G., Würtz J. and Papailiou D.D. (2000) Comparative modeling of a passive release from an L-shaped building using one, two and three-dimensional dispersion models, Int. J. Environ. Pollut., 14, 324-333.

6. Walton A., Cheng A.Y.S. and Yeung W.C. (2002) Large-eddy simulation of pollution dispersion in an urban street canyon-Part I: comparison with field data, Atmos. Environ., 36, 3601-3613. 